\title{
EDITORIAL
}

\section{The Challenge of Childhood Cancer in Developing Countries}

\author{
Abraham Haileamlak, MD, Professor of Pediatrics and Child Health
}

\section{DOI: http://dx.doi.org/10.4314/ejhs.v26i3.1}

Cancer is one of the major causes of morbidity and mortality across the world but more in developing countries. Worldwide about 160,000 new cases and 90,000 deaths occur every year in children under the age of 15 years due to cancer. Childhood cancer is the second leading cause of death worldwide surpassed only by accidents (1). Data on cancer incidence are scanty in developing countries. Most of them are hospitalbased statistics and of little value due to inadequate cancer registry and records keeping in developing countries. In developing countries, though significant progress is made in combating common childhood killers like malaria, pneumonia, gastroenteritis; cancer and trauma are emerging as major childhood killers (2). However, the greatest burden of childhood cancer is in these countries where more than $90 \%$ of the world's children are living. As stated above, over the past decades, although substantial achievements have been made in combating common communicable causes of childhood morbidity and mortality, malignancy was not considered as a public health problem in the developing world including Ethiopia. On the other hand, childhood cancers in most cases are treatable where more than $70 \%$ of these children can be cured of their cancers as demonstrated in most centers in the developed world. On the contrary, mortality rates for most pediatric cancers are close to $100 \%$ in developing countries (3).

To improve the lives of children living with cancer in the developing countries, strategies have to be designed that aimed increasing access to quality and modern treatment and define the minimal requirements for delivery of optimal care. Strategies to achieve the above goals would include the following (4):

1. Establishment of a pediatric cancer unit where needy children get access to optimum treatment; and oncology registry, research and training are coordinated.

2. Training of cancer care specialists of all cadres including nurses, clinicians, pathologists, radiologists, surgeons and other support staffs as human power is the key challenge faced by developing countries.

3. Development of standardized treatment protocols for all the major pediatric cancers as adopting protocols from the developed world may not work.

4. Establishment of supportive care and putting in place guidelines for supportive care.

5. Establishment of quality palliative care and palliative care policies.

6. Drug pricing and availability initiatives/policies.

Collaboration with international organizations and associations are paramount to the success of any national cancer program. The concept of 'twinning' in which a cancer centre from a developed country collaborates with another centre in a developing country has been successful in some countries $(5,6,7)$.

In Ethiopia, as one of the developing countries where childhood cancer is becoming one of the main public health problems, a clear treatment scheme is becoming imperative. Realizing the situation, the Federal Ministry of Health designed a national cancer control and treatment strategy. As part of this strategy, oncology treatment centers are on establishment in five teaching referral hospitals besides the only functioning center. In Parallel to this, pediatric oncology units are on establishment-one of them is the Jimma University Medical CenterPediatric Oncology/Hematology Unit (JUMC- 
POU) which is being established in collaboration with a US based ASLAN project. An inaugural workshop of this unit (May 11-12, 2016) is coincided with the third issue of the 26 volume of Ethiopian Journal of Health Sciences. This unit aspires to improve access to treatment and train all sorts of cadres required for childhood cancer treatment.

The current issue (Vol. 26, No 3), the third issue of the year contains one editorial focusing childhood cancer, eight original articles on varied subjects, one review dealing on establishment of medical schools in resource limited setting and five case reports focusing on different topics.

I invite readers to read through these articles and appreciate or utilize the contents. I also encourage readers to forward comments and suggestions.

\section{REFERENCES}

1. US Mortality Data, 2006. National Center for Health Statistics. Centers for Disease Control and Prevention, 2009.

2. Margrath I, Shad A, Epelman $\mathrm{S}$ et al. Pediatric oncology in countries with limited resources. In: Principles and Practice of pediatric oncology. Pizzo PA, Poplack DG, editors. Philadelphia: JB Lippincott Co; 1997. p.1395-420.

3. Shad A, Challinor J, Cohen ML. Paediatric oncology in Ethiopia: an inctr-USA and George Town University Hospital twinning initiative with TikurAnbessa Specialized Hospital. Cancer Control; Paediatr Cancers. 2013;108-12.

4. Lohfa B Chirdan, Fidelia BodeThomas, Oluwabunmi O Chirdan. Childhood cancers: Challenges and strategies for management in developing countries. African Journal of Pediatric Surgery, 2009: 6(2):126-130.

5. Ribeiro RC, Pui CH. Saving the ChildrenImproving Childhood Cancer Treatment in Developing Countries. N Engl J Med 2006;352:2158-60.

6. Veerman AJ, Sutaryo, Sumadiono. Twinning: A rewarding scenario for development of oncology services in transitional countries. Pediatr Blood Cancer 2005;45:103-6.

7. Pui CU, Ribeiro RC. International collaboration on childhood leukaemia. Int $\mathbf{J}$ Hematol 2003;78:383-9. 